\title{
Identification and quantification of losses in a LPT cascade by POD applied to LES data
}

\author{
D. Lengani ${ }^{\mathrm{a}, *}$, D. Simoni ${ }^{\mathrm{a}}$, R. Pichler ${ }^{\mathrm{b}}$, R.D. Sandberg ${ }^{\mathrm{b}}$, V. Michelassi ${ }^{\mathrm{c}}$, F. \\ Bertini $^{\mathrm{d}}$ \\ ${ }^{a}$ DIME Universitá di Genova, Via Montallegro 1, I-16145 Genoa, Italy \\ ${ }^{b}$ Department of Mechanical Engineering - University of Melbourne, Melbourne, Australia \\ ${ }^{c}$ GE Oil $\& 3$ Gas S.r.l. Firenze, Italy \\ ${ }^{d}$ GE AvioAero S.r.l., Torino, Italy
}

\begin{abstract}
A POD based procedure has been developed to identify and account for the different contributions to the entropy production rate caused by the unsteady aerodynamics of a low-pressure (LP) turbine blade. LES data of the extensively studied T106A cascade have been used to clearly highlight the capability of POD to identify deterministic incoming wake related modes, stochastic fine-scale structures embedded within the bulk of the wake carried during migration, and coherent structures originating in the boundary layer as a consequence of the wake-boundary layer interaction process. The POD modes computed by a kinematic kernel generate a full and complete basis, where both the velocity and enthalpy fields have been projected through an extended POD procedure to determine the relative coefficients. This allows to separately compute orthogonal sets of contributions to turbulent kinetic energy production, enthalpy-velocity correlation and turbulent dissipation
\end{abstract}

\footnotetext{
*Corresponding author

Email address: davide.lengani@edu.unige.it (D. Lengani)
} 
of resolved structures, thus clearly identifying the dominating modes (i.e. phenomena) responsible for the overall entropy production rate. Moreover, low-order truncation of these different contributions have been grouped into three different parts: those arising from the deterministic incoming wake, those due to the turbulence carried by the wakes and its interaction with the boundary layer, and those related to boundary layer events. The spatial integration of these low-order truncations restricted to the time-mean boundary layer, wake mixing and the potential flow regions of the blade passage allows gathering further information on the unsteady loss generation mechanisms, and where they mainly act. Particularly, results show that the procedure is able to decompose losses into the dominant contributions, thus providing a new tool for a rapid and clear identification of the different sources of losses in complex unsteady flow fields.

Keywords: LES, Loss production rate, POD

\section{Introduction}

The development of the flow field in turbine and compressor rows of multistage turbomachines is a complex three dimensional and unsteady phenomenon. The wakes shed from upstream rotating blades induce periodic (deterministic) and stochastic oscillations at the entrance of the downstream rows, while the potential field induces additional time-varying flow distortion, making the propagation of turbulent quantities generated by upstream wakes strongly inhomogeneous and spatially and temporally dependent. During migration the upstream wakes diffuse in the blade rows gaps and in the downstream passage, they give rise to turbulent and viscous losses due to 
superposition of different phenomena. The identification and quantification of the various contributions to the overall loss remain a difficult task, as also pointed out by van de Wall et al. (2000); Praisner et al. (2006).

In the last years detailed high fidelity computations and accurate experiments (see Wu and Durbin 2001; Michelassi et al. 2003; Stieger and Hodson 2005; Hodson and Howell 2005 for example) have given deep insight into the unsteady wake migration process. Approaching the downstream cascade, the planar-like (2-D like) structure of the incoming wake undergoes strong bowing, tilting, dilatation and stretching processes, and it finally becomes fragmented into segments as a consequence of the relative motion between the stator and rotor rows (Binder et al., 1985, 1989).

The Direct Numerical Simulation (DNS) of Wu and Durbin (2001) described in detail the stretching, dilatation and compression processes of wake filaments evolving in a LP turbine passage through a strain rate tensor principal axes analysis. They clearly showed that the wake filament developing close to the pressure side is almost aligned with the stretching direction, while on the other hand the wake centerline at the bow apex is mainly aligned with the compression direction. Moreover, as also shown in Michelassi et al. (2003), the largest eigenvalues are localized on the suction side of the channel, where the strongest reorientation of the jet-like structure takes place. The eigenvalue and eigenvector decomposition of the shear and strain tensors reported in Michelassi and Wissink (2015) shows that the largest (compression related) strain eigenvalues dominate the turbulent kinetic energy production due to wake migration, with a maximum in the flow region where stress and strain principal axes are almost aligned. These observations agree well with 
Rogers (2002) who discusses the effects of different strain magnitudes and orientations on the turbulence produced in a planar wake. He clearly highlights that the condition with the wake axis aligned with the eigenvector of the velocity gradient tensor associated with compression represents the most influencing for turbulence production, similar to what happens in the bow apex of the wake migrating through the LPT passage (see also Stieger and Hodson 2005).

The incoming wakes are also responsible for transporting the finer structures embedded in the bulk of the wake into the suction side boundary layer, further contributing to the excitation of the boundary layer and the consequent excitation of the transition process (see Wu et al. 1999; Lengani et al. 2017b). The isocontour surfaces of the $\lambda_{2}$ criterion and instantaneous flow vorticity reported in Zaki et al. (2009) and Sarkar and Voke (2006) give a clear view of the finer structures carried by the wakes. These structures are responsible for the generation of a dense population of streaky structures in the footprint of the region perturbed by wakes, as observed both numerically or experimentally (Wu et al. 1999; Nagabhushana Rao et al. 2013; Coull and Hodson 2011; Lengani et al. 2017b). Moreover, for highly loaded turbine blades the boundary layer growing between successive wakes may undergo an intermittent separation (depending on the loading and diffusion level (Satta et al., 2014), that evidently imposes an inviscid-like instability (Sarkar and Voke, 2006; Simoni et al., 2013). In this case large scale coherent structures are shed as a consequence of the separated shear layer rollup during wakeboundary layer interaction, with dynamics similar to those characterizing the separated flow transition mechanisms observed in cases without incom- 
ing wakes (Alam and Sandham, 2000; Jones et al., 2008).

Overall, the unsteady aerodynamics of a LP passage is the result of the superposition of different phenomena acting in the different parts of the passage with different temporal and spatial scales. Each of them differently affects the loss (i.e. entropy) production mechanisms in the entire downstream passage (i.e. inside and outside the blade boundary layers) that up to now have usually been evaluated as a whole. The possibility to split and detect the different sources of losses represents one of the main engineering goals for further increasing the efficiency of modern new generation LPT blades. Optimization procedures aimed at selectively reducing losses could be easily and efficiently implemented observing the effects of design parameters on the different loss sources, instead of the effect on the overall loss. The entropy rate of change split allows determining the loss contribution due to wake diffusion in the downstream passage and differentiate it from those being generated as a consequence of the wake boundary layer interaction, due to both large and finer scales carried during wake migration. Due to the aforementioned (turbulent) stress-strain interaction mechanisms, wake migration in the potential flow region of the downstream passage gives rise to deterministic stress production, following the terminology reported in van de Wall et al. (2000), that can contribute a significant portion of energy degradation. The identification of this additional loss source will also be important for correcting RANS predictions of multistage machines, since in this numerical method the wake shear-stress interaction process producing losses is not resolved. Michelassi et al. $(2015,2016)$ used DNS and LES to show that entropy generation due to wake migration represents a non-negligible 
contribution to overall losses. The different sources of losses were quantified by a control volume method (following Denton 1993) and not directly quantified from the dataset due to the difficulty in separating boundary layer from wake migration related events. Neither phase-averaged (Hussain and Reynolds, 1970) nor generalized phase-averaged methods (Bourgeois et al., 2013) can achieve such a separation, since all phenomena not directly locked with the passing wake forcing are smeared out by the averaging operation and cannot be directly resolved.

Among other data analysis approaches, Proper Orthogonal Decomposition (POD), that was introduced by Lumley (1967), is now a mature approach that has been often used to identify the different dynamics driving complex turbulent flows (e.g. Liu et al. 2001; van Oudheusden et al. 2005; Perrin et al. 2007; Kurelek et al. 2016). However, it has rarely been applied to the analysis of turbine blades perturbed by unsteady wakes. In the work of Sarkar (2008), POD was applied to LES data describing the unsteady flow field of the T106 turbine cascade with $\operatorname{Re}=160,000, f^{+}=0.68$. Here, the author paid attention to the dynamics through which the large scale deterministic structures attached to the leading and trailing boundaries of the incoming wakes interact with the separated shear layer growing between wakes. In Lengani et al. (2017b), POD was applied to experimental data in order to discuss the flow physics of the transition induced by the periodic passing wakes. It has been shown that POD can be used to identify the large scale structures of the passing wake, as well as observing the effect of the turbulence carried by the wake and its effects on the boundary layer.

In the present paper, POD has been applied to high fidelity Large Eddy 
Simulation (LES) data of the T106 LPT cascade with unsteady incoming wakes generated by a set of moving bars to mimic a stator-rotor interaction. The aim is to determine if the POD applied to LES data can identify and discern the different loss mechanisms in the presence of unsteady incoming wakes. This is accomplished by a new POD based procedure, that follows the idea reported in Lengani et al. (2017a), that allows the identification and characterization of the different dynamics that contribute to loss generation, analyzing their contribution to the entropy rate of change. The bi-orthonormality condition of the POD modes and related temporal coefficients is considered to compute the contribution of the individual mode dynamics to the Reynolds stresses and hence to rate of change of total pressure. An extended POD projection (Borée, 2003) on the kinematic basis also allows computing the rate of change of total enthalpy, thus the possibility to account for single mode contribution to the entropy rate of change. Losses generated in the boundary layer region as a consequence of streaky structures and wake-boundary layer interaction mechanisms are clearly captured and separated from losses due to wake migration and distortion in the potential flow region due to both large and finer scales.

The paper is organized as follows: in $\S 2$ the numerical method, the geometrical configuration and the flow parameters are described in detail; in $\S 3$ the basic equations and the decomposition procedure for the moment statistics are presented; in $\S 4$ results from POD of the LES data are described in order to clearly identify the different dynamics acting in the different regions of the LP turbine passage; once recognized, in $\S 5$ a limited number of POD modes has been used for the identification of the contributions to the entropy 


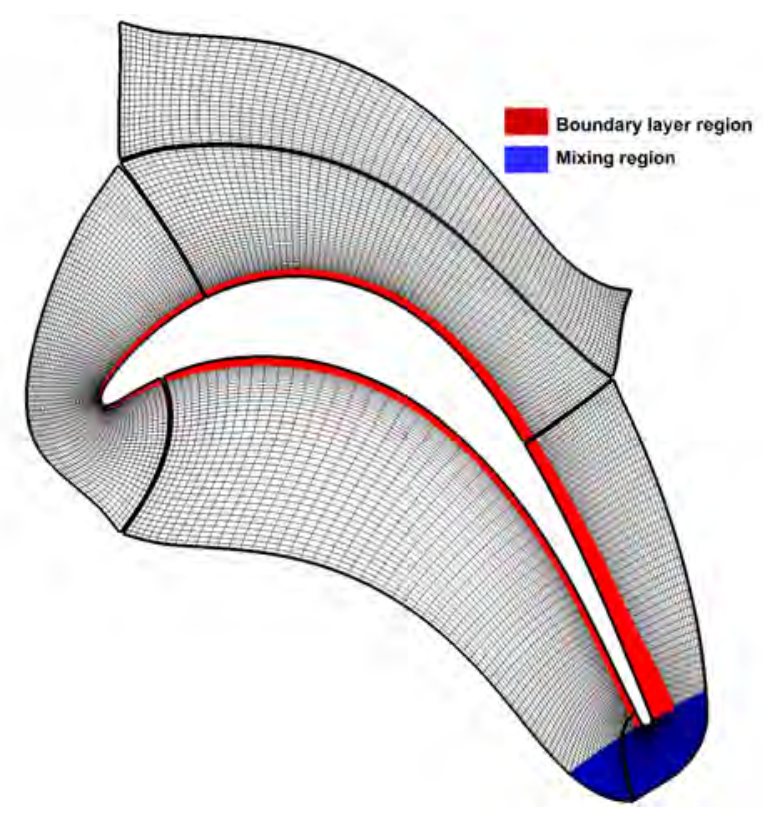

Figure 1: Scheme of the computational domain and integration areas.

rate of change due to wake migration and boundary layer related processes. Finally, in $\S 6$ spatial integration in the different parts of the channel allows a quantitative analysis of the different sources causing an entropy change in the different regions.

\section{Large Eddy Simulations}

The low-pressure turbine data-set analyzed in this paper was obtained by performing large eddy simulations (LES) using the in-house compressible multi-block structured Navier-Stokes solver HiPSTAR. A comprehensive description of the algorithm and validation for turbomachinery applications is given in Sandberg et al. (2015) and here only a brief summary of the key features is presented. The code uses fourth-order accurate wavenumber optimized compact finite differences (Kim and Sandberg, 2012) in the 
axial/pitchwise plane and a pseudo-spectral approach, using the FFTW library, in the homogeneous spanwise direction. For the advancement in time an ultra-low storage five-step explicit fourth-order Runge-Kutta method was used (Kennedy et al., 2000). To increase the robustness of the numerical scheme and to avoid aliasing errors of the cubically nonlinear convective terms within the discretized Navier-Stokes equations, a skew-symmetric splitting of the non-linear terms according to Kennedy and Gruber (2008) was employed.

The code has been thoroughly tested on a range of canonical flows, such as turbulent pipes and sub- and supersonic instability waves, and has been shown to accurately reproduce the flow in linear low-pressure turbine cascades (Sandberg et al., 2015), and, more recently, high-pressure turbine cascades (Wheeler et al., 2016). More recently, LES models have been implemented into the code to reduce computational cost and allow for parametric studies of turbine flows, and the suitability and accuracy of the WALE model (Nicoud and Ducros, 1999) was demonstrated in another LPT study that investigated the concerted action of reduced frequency and flow coefficient on unsteady loss generation (Michelassi et al., 2016). The numerical set-up for the low-pressure turbine cases conducted to provide data for the POD analysis follows that described in Michelassi et al. (2015). A linear low-pressure turbine cascade using the T106A profile was considered at the isentropic conditions $R_{2 i s}=60,000$ and $M_{2 i s}=0.4$, based on axial chord and isentropic exit velocity $V_{2 i s}$. The grid topology comprised four blocks for an O-type grid around the blade to ensure optimal grid quality in the vicinity of the wall, and five blocks for an H-type grid allowing for pitchwise periodicity. To ensure high-quality solutions at the block interfaces despite metric 
discontinuities, all blocks were connected to each other using characteristic interface conditions. At the wall a no-slip isothermal boundary condition was used. To generate periodically incoming bar wakes, an immersed boundary method was used, placing cylinder bars with diameter $D / C_{a x}=0.02$ at $x / C_{a x}=-0.7$, moving in the pitchwise direction with velocity $V_{b a r}$. By varying the bar spacing and/or $V_{b a r}$ the reduced frequency, $F_{r e d}=\frac{f \cdot C_{a x}}{V_{2 i s}}$, could be varied, where $f$ is the bar passing frequency. The data analysed in this paper were obtained with $V_{b a r}=-0.41 U_{\infty}$, where $U_{\infty}$ is the incoming freestream velocity, resulting in $F_{\text {red }}=0.61$. The simulation was initially run for 5 flow-through times in 2D, then 3D simulations were restarted from the fully developed 2D result. After about 10 additional flow-through times, to avoid the transient period, 900 snapshots were collected for 15 bar passing periods for the present POD analysis.

\section{Fundamental equations}

The different terms involved in the entropy rate of change are first briefly introduced in order to be able to then focus on the more relevant quantities to be decomposed in the following sections. Following Gibbs' equation, for a 3-D non stationary flow the entropy variation over time $(D s / D t)$ can be expressed as a function of total quantities (temperature $T_{t}$, enthalpy $h_{t}$, density $\rho_{t}$ and pressure $\left.p_{t}\right)$ :

$$
T_{t} \frac{D s}{D t}=\frac{D h_{t}}{D t}-\frac{1}{\rho_{t}} \frac{D p_{t}}{D t}
$$

Since the aerodynamic efficiency of LPT blades is definitively described by the mean entropy flux variation, we are interested in long time averaged 
quantities. Following a Reynolds averaging approach, the balance equation for the time-mean total enthalpy (e.g.,Leschziner 2015) can be formulated as follows:

$$
\frac{D \bar{h}_{t}}{D t}=\frac{\partial}{\partial x_{i}}\left[\kappa\left(\frac{\partial \bar{h}_{t}}{\partial x_{i}}\right)-\overline{u_{i}^{\prime} h_{t}^{\prime}}\right]+\nu \overline{\left(\frac{\partial u_{i}^{\prime}}{\partial x_{j}}+\frac{\partial u_{j}^{\prime}}{\partial x_{i}}\right) \frac{\partial u_{i}^{\prime}}{\partial x_{j}}}
$$

where $\kappa$ is the thermal conductivity. While, the balance equation for the time-mean total pressure (e.g., Hanjalić and Launder 2011) reads as:

$$
\frac{D \bar{p}_{t}}{D t}=\rho \overline{u_{i}^{\prime} u_{j}^{\prime}} \frac{\partial U_{i}}{\partial x_{j}}-\rho \nu\left(\frac{\partial U_{i}}{\partial x_{j}}\right)^{2}+\rho \nu \frac{\partial^{2} K}{\partial x_{j}^{2}}-\frac{\partial\left(U_{i} \overline{u_{i}^{\prime} u_{j}^{\prime}}\right)}{\partial x_{j}}
$$

where $K$ is the kinetic energy. Since the volume integral of the diffusive terms is negligible, the balances can be expressed as follows (equations are written now in the non-dimensional form):

$$
\begin{gathered}
\frac{D \bar{h}_{t}}{D t}=\underbrace{-\frac{\partial \overline{u_{i}^{\prime} h_{t}^{\prime}}}{\partial x_{i}}}_{I}+\underbrace{\frac{1}{R e_{\infty}} \overline{\left(\frac{\partial u_{i}^{\prime}}{\partial x_{j}}+\frac{\partial u_{j}^{\prime}}{\partial x_{i}}\right) \frac{\partial u_{i}^{\prime}}{\partial x_{j}}}}_{I I} \\
\frac{D \bar{p}_{t}}{D t}=\underbrace{\rho \overline{u_{i}^{\prime} u_{j}^{\prime}} \frac{\partial U_{i}}{\partial x_{j}}}_{I I I}-\underbrace{\frac{\rho}{R e_{\infty}}\left(\frac{\partial U_{i}}{\partial x_{j}}\right)}_{I V}
\end{gathered}
$$

Substituting eqs. 4 and 5 in eq. 1 and applying it to a finite control volume of fluid elements covering one entire cascade pitch, the following balance equation can be obtained after some algebra:

$$
\tilde{s}_{2}-\tilde{s}_{1}=\frac{1}{\dot{m}}\left(\int_{V} \frac{(I+I I)}{T_{t}} d V-\int_{V} \frac{(I I I+I V)}{T_{t} \rho_{t}} d V\right)
$$

where $\tilde{s}_{i}$ denotes the mass-averaged inlet and outlet cascade entropy per unit mass. The first term on the right hand side provides the entropy rate of 
change due to the enthalpy-velocity correlation terms (i.e. the vector of the scalar stresses) and the fine (resolved) turbulent scales' dissipation producing heat. The contribution due to mechanical energy dissipation comes from the second term on the right hand side. Here viscous and turbulent stresses produce entropy. All the other diffusive-like terms appearing in Eqs. 2 and 3 do not contribute to the balance described by Eq. 6, since their spatial integral values are zero. Moreover, contributions due to the unresolved scales will contribute to the balance, especially due to dissipation. However, it has been shown in Michelassi et al. (2016) that the present LES results match the DNS one with a reasonable accuracy. Hence, only minor inaccuracies in the quantitative estimation reported in the last section of the paper are expected.

\subsection{Data processing}

In the present work, all fluctuating quantities are decomposed in order to separate the different dynamics and analyze their contribution to the rate of change of entropy. POD of the flow field is chosen as a suitable tool for such a loss split. The POD has been computed following the snapshots method of Sirovich (1987) constructing snapshots from the velocity field. This decomposition provides a triplet of information: the eigenvalues $\lambda^{(k)}$, the eigenvectors $\chi^{(k)}$ and the POD modes $\phi^{(k)}$. The eigenvalue of the $k^{\text {th }}$ mode represents the energy contribution of the mode to the total kinetic energy of velocity fluctuations. The eigenvectors constitute an orthogonal basis and retain the temporal information related to each mode. The POD modes constitute an orthogonal basis that provides the spatial information identifying coherent structures in the flow. 
The terms I, II and III of eq. 6, involved in the entropy rate of change, are due to the turbulence activity, being defined by fluctuating quantities. The contribution per POD mode of each of these terms can be efficiently computed exploiting the properties of POD. Particularly, since the POD modes and eigenvectors are orthogonal, the Reynolds shear and normal stresses can be computed as follows:

$$
\overline{u_{i}^{\prime} u_{j}^{\prime}}=\sum_{k} \phi_{u_{i}}^{(k)} \phi_{u_{j}}^{(k)}
$$

The term $\phi_{u_{i}}^{(k)} \phi_{u_{j}}^{(k)}$ represents the contribution of the $k^{t h}$ POD mode to the overall time-mean Reynolds stresses. This property of POD can be applied to determine the individual mode contribution to the TKE production (the term III in Eq. 6) with reversed sign, that can be generically represented (using index $i, j$ ) as $p_{i j}$ :

$$
p_{i j}=-\overline{u_{i}^{\prime} u_{j}^{\prime}} \frac{\partial U_{i}}{\partial x_{j}} .
$$

Substituting Eqs. 7 into Eq. 8, the contribution of each POD mode to the TKE production rate $p_{i j}$ can be written as:

$$
p_{i j}^{(k)}=-\phi_{u_{i}}^{(k)} \phi_{u_{j}}^{(k)} \frac{\partial U_{i}}{\partial x_{j}}, \quad \text { with } \quad p_{i j}=\sum_{k} p_{i j}^{(k)} .
$$

According to this equation, each POD mode contributes to a quota of the time-mean TKE generation rate, explicitly appearing in Reynolds averaged formulation of the Navier-Stokes equations. As shown previously in Lengani et al. (2016), a combination of POD modes of the velocity field and their spatial derivatives can be used to obtain the dissipation of energy due to the resolved scales (the term II into Eq. 6). It is expressed as:

$$
\frac{1}{R e_{\infty}} \overline{\left(\frac{\partial u_{i}^{\prime}}{\partial x_{j}}+\frac{\partial u_{j}^{\prime}}{\partial x_{i}}\right) \frac{\partial u_{i}^{\prime}}{\partial x_{j}}}=\frac{1}{R e_{\infty}} \sum_{k}\left(\frac{\partial \phi_{i}^{(k)}}{\partial x_{j}}+\frac{\partial \phi_{j}^{(k)}}{\partial x_{i}}\right) \frac{\partial \phi_{j}^{(k)}}{\partial x_{i}} .
$$


The enthalpy-velocity correlation term (term I of Eq. 6) can be expressed as:

$$
-\frac{\partial \overline{u_{i}^{\prime} h_{t}^{\prime}}}{\partial x_{i}}=-\sum_{k} \frac{\partial \phi_{u_{i}}^{(k)} \phi_{h_{t}}^{(k)}}{\partial x_{i}}
$$

where the $k^{t h}$ POD mode of the enthalpy $\phi_{h_{t}}^{(k)}$ is obtained by projecting the instantaneous enthalpy fields $h_{t}^{\prime}$ onto the orthogonal basis spanned by the kinetic POD eigenvector $\left(\chi^{(k)}\right)$ of the velocity correlation tensor, like the extended POD procedure proposed by Borée (2003):

$$
\phi_{h_{t}}^{(k)}=\sum_{n=1}^{N} \chi_{n}^{(k)} h_{t n}
$$

where $n$ is the discrete time interval. This last equation can be used to provide a formal proof of eq. 11 (omitted for brevity). According to these equations, each POD mode contributes a part of the total entropy generation, which can be analyzed separately in order to identify the role it plays in the different dynamics affecting the unsteady flow field of the LPT cascade. The term IV of Eq. 6 is the viscous dissipation due to the time-mean flow distortion that accounts for a large portion of the overall losses (about $40 \%$ ), it is concentrated in the boundary layer and profile wake mixing regions, as expected, with negligible contribution in the potential flow region of the blade passage affected by incoming wake migration. Since this term is not dependent on the dynamics and cannot be decomposed, it will not be further discussed in the following but only accounted for in the cumulative distributions reported at the end of the paper.

The 900 instantaneous snapshots adopted to compute POD assure a good convergence of the results. The error in the computation of the relative TKE 
captured by the most energetic modes (the first 30 ) is below $0.5 \%$, in fact POD reaches a statistical convergence on the first 10 modes for more than 200 data, see for example Lacarelle et al. (2009). The computation of the higher order POD modes has a slightly higher error, but this error can be minimized summing up their contribution to TKE or entropy rate of change when considering families of modes that correspond to different dynamics (as discussed in section 4.4).

\section{Results}

\subsection{Instantaneous Flow Structures}

The instantaneous flow field of the LES is depicted in Fig. 2 for two typical snapshots within a wake passing cycle in order to give a qualitative view of the fluctuating field and its complexity. The flow field is viewed at the mid-span section of the simulation and is represented with vectors of the fluctuating velocity and iso-contours of the fluctuating pressure (i.e. velocity and pressure are decomposed with the Reynolds' decomposition, subtracting the mean value). In the first frame the instantaneous velocity vectors point, in the mid-section of the passage, towards the suction side of the bottom blade. They unveil the remnants of the wake "centerline" and this flow region is evidently characterized by negative relative pressure (blue area in the plot). Disordered motion can also be recognized in the surrounding of the blade leading edge, as well as in the rear part of the blade suction side. At the leading edge the pressure field shows localized spots at elevated pressure. The snapshot at a later time instant, where the wake centerline is leaving the simulation domain, shows a complex pattern in both the potential flow 

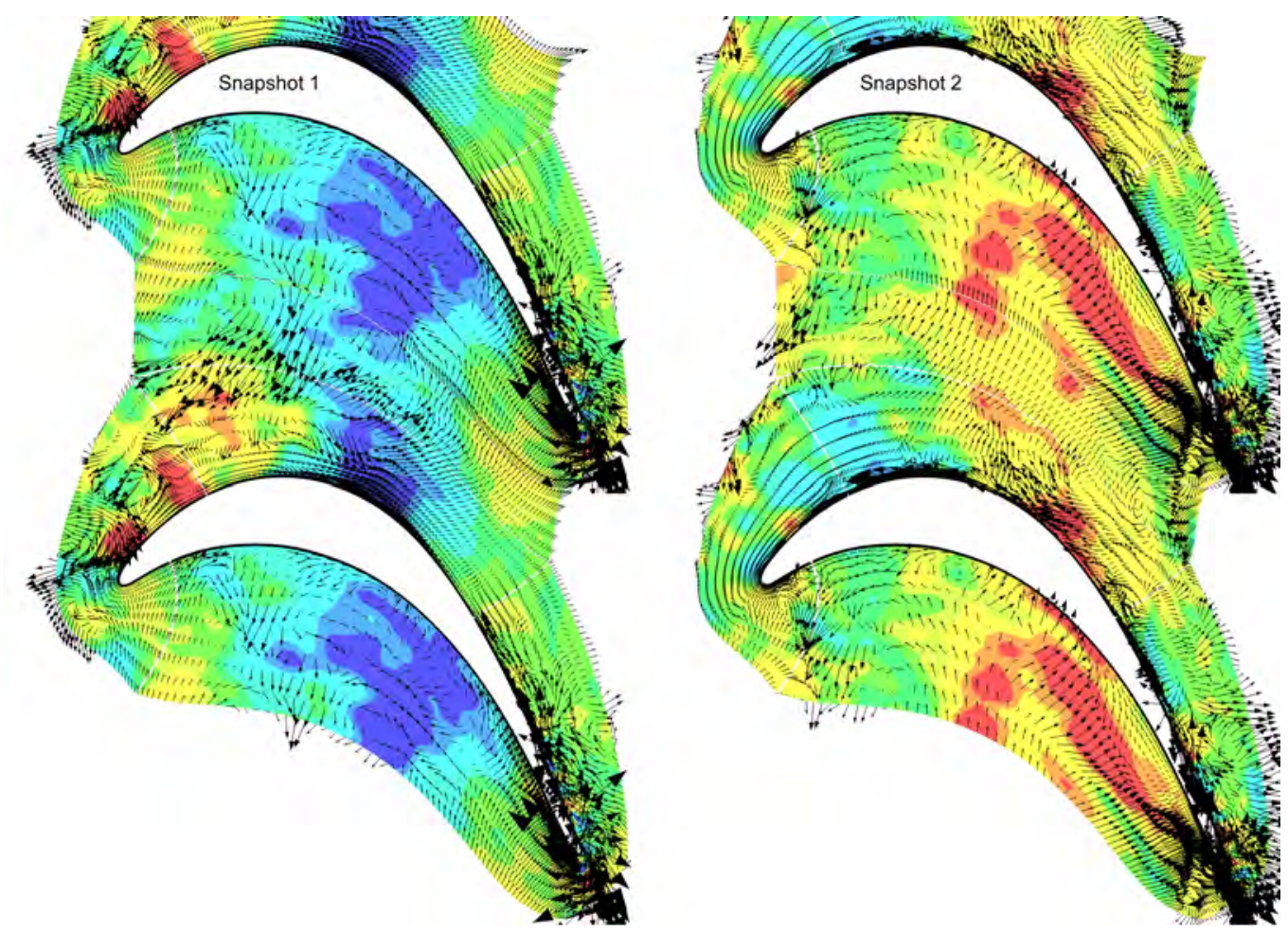

Figure 2: Instantaneous perturbation velocity vector plots and pressure fluctuations for two different time instants of the blade passing cycle. Blue and red contours represent minimum and maximum of pressure fluctuations (i.e. mean subtracted), respectively.

region and in the proximity of the wall. Now the instantaneous velocity vectors point away from the suction side wall at around 0.5 of axial chord downstream of the leading edge, and locally the pressure increases. At this time instant the structures carried by the upstream wake towards the suction side boundary layer are interacting with the rear part of the blade.

The structures originating from this interaction are highly three dimensional as shown in the iso-contours of the pressure fluctuations depicted in Fig. 3. The aft portion of the suction side is depicted in the $3 \mathrm{D}$ view of this picture for the two different time instants shown in the previous plots. In 


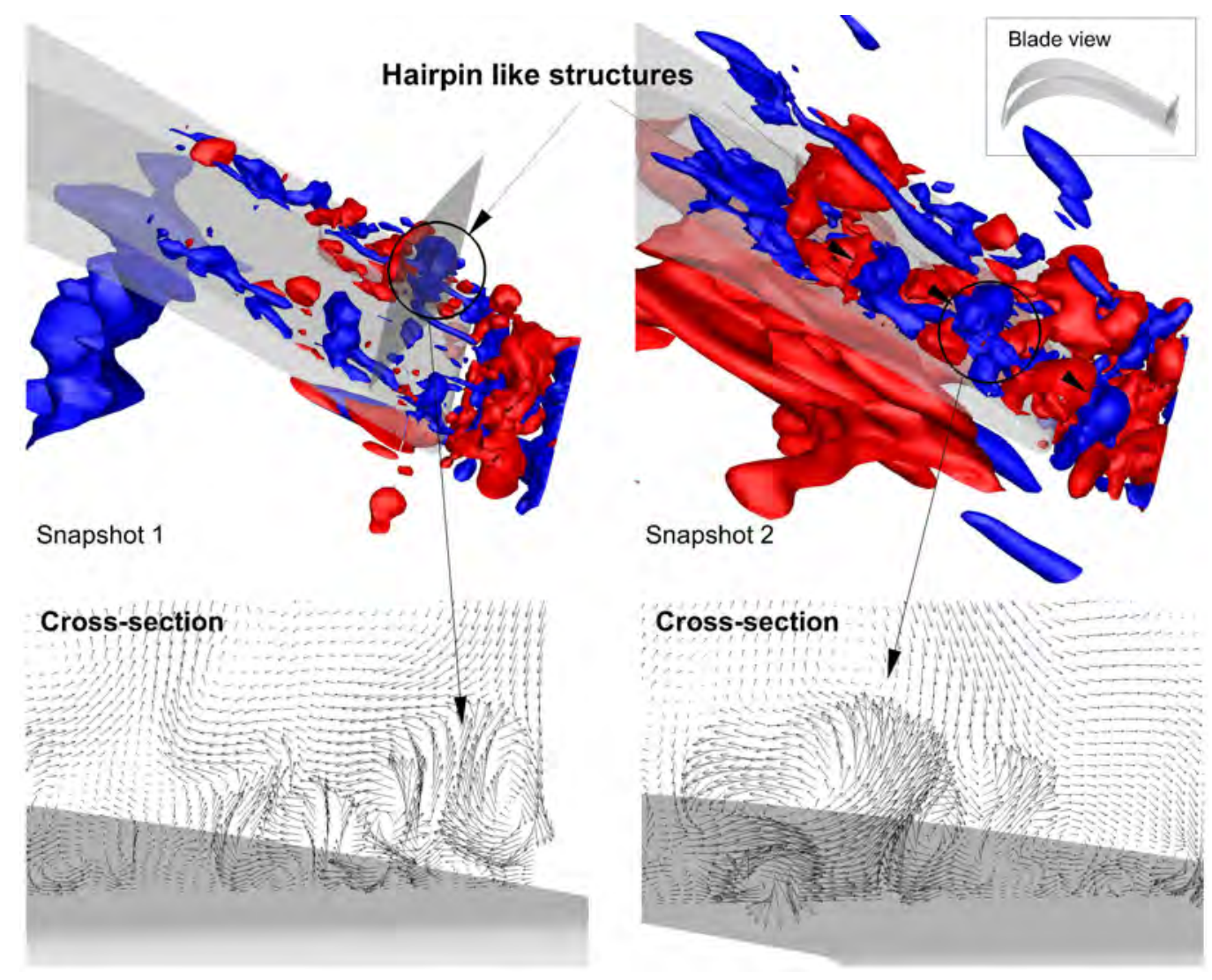

Figure 3: On top: 3D view of the instantaneous pressure fluctuations for two different time instants of the blade passing cycle. Blue and red iso-surfaces represent pressure fluctuations below and above a reference value, respectively. On bottom: instantaneous perturbation velocity vector in a cross-stream plane perpendicular to the blade surface (as identified from the sketch of the top right corner). 
the first snapshot the wake is not yet affecting the rear portion of the suction side, and a scarce population of coherent structures can be observed. Particularly, only small scale structures appear to be present over this portion of the blade at this time. These structures grow within the boundary layer and are not related to the wake-boundary layer interaction process (since at this phase the wake is not interacting with this flow region of the suction side). The cross-stream plane perpendicular to the blade surface on the bottom of Fig. 3 helps the visualization of such structures (as observed by Michelassi et al. 2003); counter rotating vortices that represent the legs of hairpin vortices are identified (similar to those observed also in Adrian 2007). In the second snapshot, the flow shows a dense population of structures that are typically observed in turbulent flows, such as a train of hairpin vortices (that may be identified with a minimum of pressure as well as in the cross-stream plane) and boundary layer streaks. These structures are forced by the wake turbulence, as was already shown in Wu et al. (1999); Nagabhushana Rao et al. (2013); Coull and Hodson (2011); Lengani et al. (2017b), and are representative of the bypass-type transition process periodically induced by the passing wakes (Dick and Kubacki, 2017).

\subsection{POD analysis}

The instantaneous LES flow fields are highly complex, and a technique is required to rigorously identify the different dynamics responsible for loss generation. As a first step, POD is adopted in order to provide a statistical representation of the deterministic and stochastic contributions to the velocity fluctuations. The eigenvalues $\lambda$ provide the kinetic energy captured by the POD modes, and give the first information of the relative importance of 


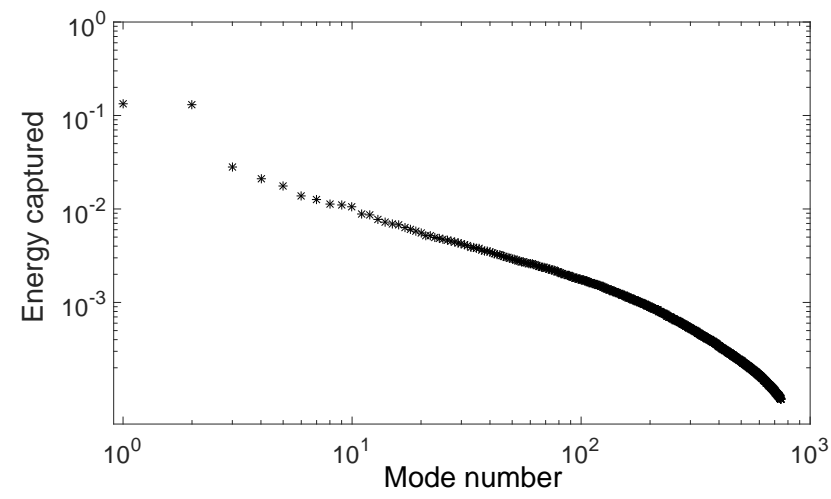

Figure 4: POD eigenvalues.

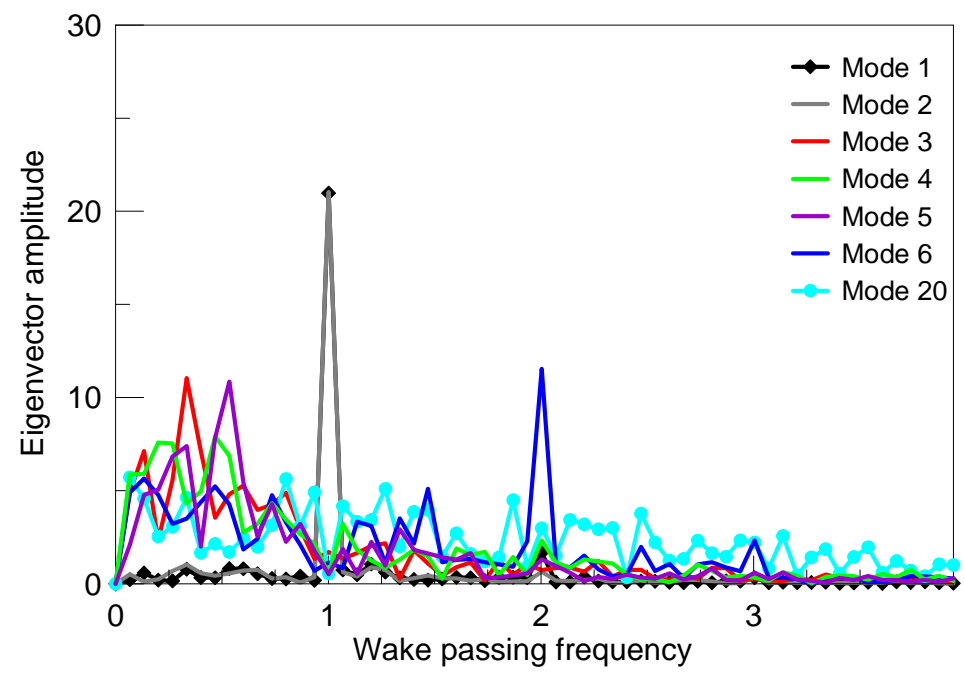

Figure 5: Spectral analysis of the POD eigenvectors. 
each mode. Figure 4 shows the POD eigenvalues normalized with the total kinetic energy. The first two modes have a total contribution of about $25 \%$ of the total energy, while the energy of the modes above the $100^{\text {th }}$ becomes lower than $0.1 \%$. Even though the energy distribution seems rather similar for the modes from 3 to 10, the temporal and spatial distribution of the POD modes highlights different physical mechanisms involved in the generation of velocity fluctuations.

In order to better distinguish between the different dynamics captured by the modes, a Fourier analysis of the POD eigenvectors is also conducted and the results are shown in Fig. 5. The frequency resolution of this analysis is $1 / 15$ th of the wake passing frequency, that is sufficient to provide the frequency range of interest of the different phenomena. The first two modes are strongly related to the wake passing frequency, identifiable as a sharp peak in the spectrum at that frequency. The modes 3 to 5 have high amplitude at low frequencies and, interestingly, a negligible contribution at the wake passing frequency and its higher harmonics. Conversely, the following modes, such as the $6^{\text {th }}$, are again related to the wake passing frequency since they clearly exhibit higher harmonics of the fundamental frequency. Modes above 12 , as for example mode 20 depicted in the figure, are no more related to the wake passing frequency or to the low frequency events, since its spectrum evidently exhibits a broadband behaviour.

Some of these POD modes, that exhibit different characteristics, have been opportunely selected and shown in Fig. 6 as vectorial representations: each vector component is provided by the POD mode of the corresponding velocity component. The POD modes are orthonormal, thus the vector 
length for the different modes does not provide a direct comparison of their contribution to the velocity fluctuations. To this end the isocontour plot represents the spatial distribution of the normalized TKE captured by the mode. These quantities are also represented in Fig. 7 that provides a 3D view of the rear part of the blade, where the isocontours with values close to the maximum levels are reported. It is used to highlight where the main flow features are identified and their spatial scales, while the next section (sec. 4.3) will discuss how and where the losses are produced.

The first two, most energetic, POD modes identify the large scale vortices that are associated with the deterministic "jet-like" structure induced by the wake (Sarkar and Voke, 2006; Lengani et al., 2017b). Here, only the first mode is reported for the sake of brevity, since the second one basically depicts the same flow structures with the same energy content and frequency (see Figs. 4 and 5) but spatially shifted. This behaviour is typical for pseudoperiodic convective flows (Legrand et al., 2011a,b; Yarusevych and Kotsonis, 2017; Wen et al., 2016). As discussed in a previous publication (Lengani et al., 2017b), these modes can be used to reconstruct the phase-averaged flow field and describe the migration of the deterministic part of the wake traveling across the turbine passage, similarly to the generalized phase-averaged procedure described in Bourgeois et al. (2013) to reconstruct the coherent motion behind a square cylinder. The structure represented by these first two modes divides into two branches; two counter-rotating large scale vortices can clearly be identified in the pictures (highlighting the "negative-jet" like structure described in Stieger and Hodson 2004). Interestingly, the maximum TKE can be observed where the counter-clockwise rotating structure 

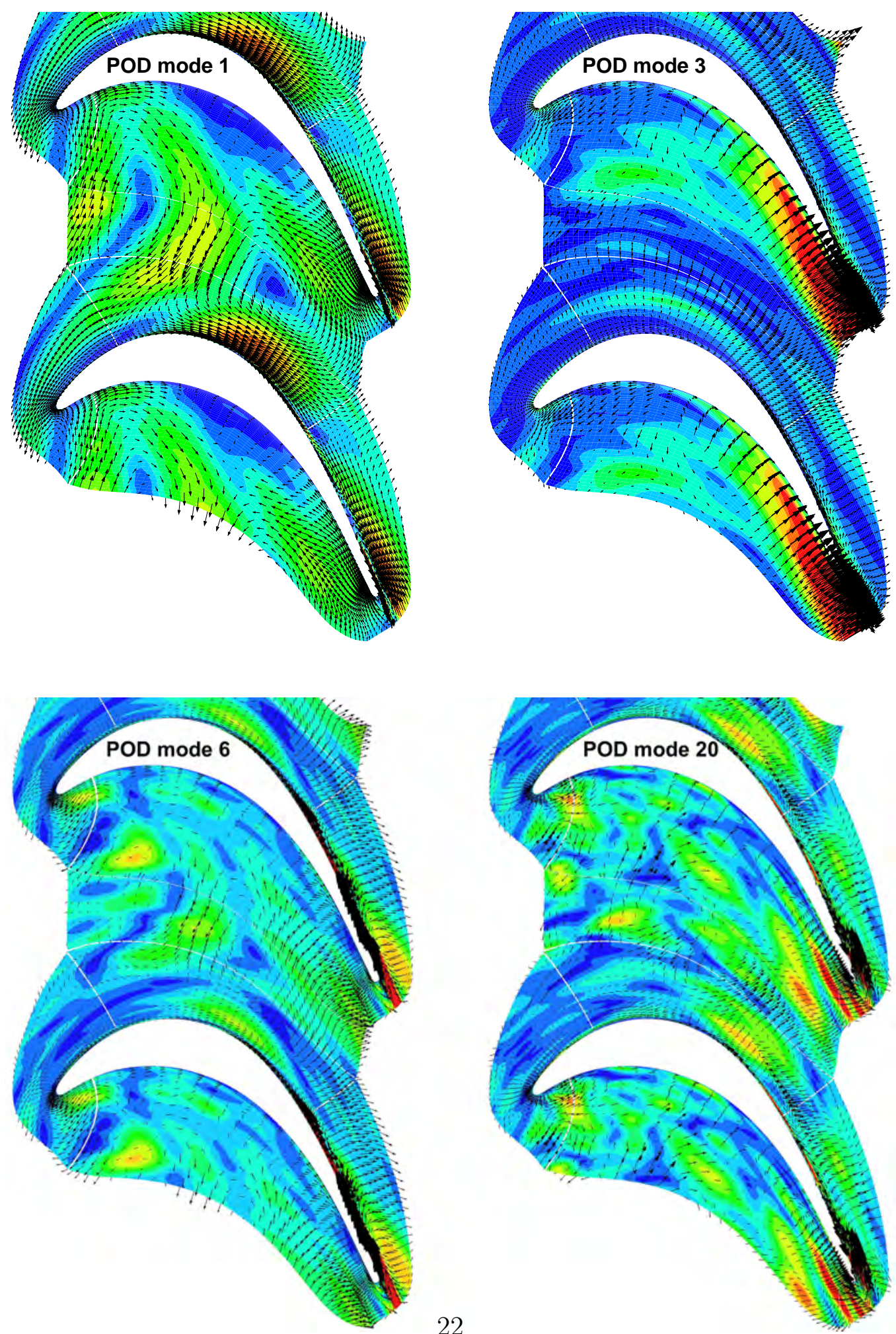

Figure 6: Vectorial representation of POD modes and spatial distribution of the TKE captured by the respective POD mode. Red and blue color represent high and low TKE, respectively 

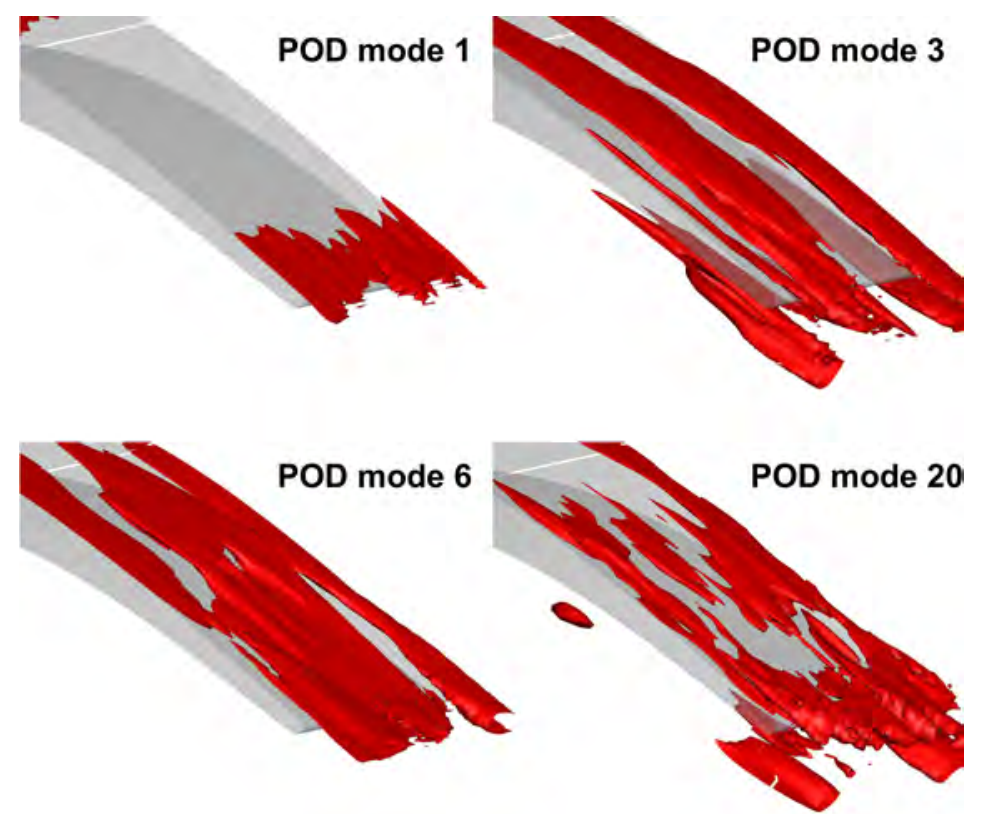

Figure 7: Isosurface of the TKE captured by individual POD modes.

(the leading boundary of the wake) interacts with the blade suction side, just behind the peak suction position (see mode 1, where the local TKE peak is the result of the wake distortion). High values of TKE can also be observed close to the trailing edge (see also higher modes, suggesting this second peak could be attributed to a local boundary layer transition). The 3D view of Fig. 7 shows evidence that these wake related modes have a strong spanwise coherence.

Mode three is related to flow oscillations that occur in the rear part of the blade. The highest energy of these fluctuations is located on the blade pressure side, where typically Görtler vortices are present. The authors suspect that the low frequency motion of the streamwise vortex filament (as those observed in Michelassi et al. 2003) affecting the rear part of the blade pres- 
sure side is responsible for the quite large area traced by this mode. Indeed, it is worth noting that a single POD mode does not represent a structure, but more properly it provides a view of its best statistical representation in terms of its contribution to flow oscillations. The fluctuating energy of this mode is particularly low within the blade passage and hence mode 3 seems to not be related to the upstream wake migration process. Furthermore, the $3 \mathrm{D}$ view of Fig. 7 makes clear that, conversely to mode 1 and mode 2, these fluctuations appear as large filaments elongated in the streamwise direction and hence probably related to three-dimensional large scale structures.

The other modes depicted in the figures (modes 6 and 20) show coherent structures in the LPT passage similar to modes 1 and 2, but they also exhibit significant TKE levels in the rear part of the blade suction side. Having observed their structure in proximity to the wall, it appears reasonable to state that both these modes are likely to represent the unsteady interaction between the wake and the boundary layer. Indeed, these modes are three dimensional, being elongated in the streamwise direction as depicted in Fig. 7, and they provide a statistical representation of the flow field that resembles the streaky structures induced by the periodic by pass-type transition process previously observed in the instantaneous images. Hence, the wake passing over the blade and interacting with the boundary layers leads to the generation of 3D structures and regions with high and low velocities within the boundary layer. However, even though their structure into the boundary layer looks similar they represent different dynamical features. In fact, mode 6 is related to the deterministic blade passing events according to the FFT analysis of the eigenvectors, since it clearly exhibits a peak at the higher 
harmonic of the fundamental frequency. Mode 20 is instead not related to the wake passing frequency, and shows a broad band shape. Hence, mode 6 represents deterministic events induced by the large scale structures of the passing wake (in the passage and in the boundary layer), while mode 20 is a statistical representation of the turbulence carried by the bulk of the wake in the potential flow region, and the boundary layer streaks forming during the by pass-like transition process.

Results presented in this section therefore illustrate that POD modes defined by the kinematic kernel are able to identify and split the different dynamics related to wake or boundary layer events affecting the unsteady flow field characterizing the LPT passage. Moreover, by analyzing the POD mode distributions jointly with the spectral content presented here, it is also possible to associate families of modes with particular dynamics (i.e. related to large scale or finer structures carried by the wake in both the potential flow and boundary layer regions).

\subsection{TKE production}

As described in the previous sections the overall entropy rate of change (Eq. 6) is comprised of the sum of the effects related to TKE production, heat dissipated by the resolved scales, velocity-enthalpy correlation and viscous dissipation due to mean flow distortion. The fourth term, as previously discussed, cannot be decomposed on a modal basis. The first three terms are, instead, analyzed separately, focusing on the contributions due to the different POD modes. Particularly, the TKE production, that is the main contribution to the loss generation, is analyzed in details in this section, while 

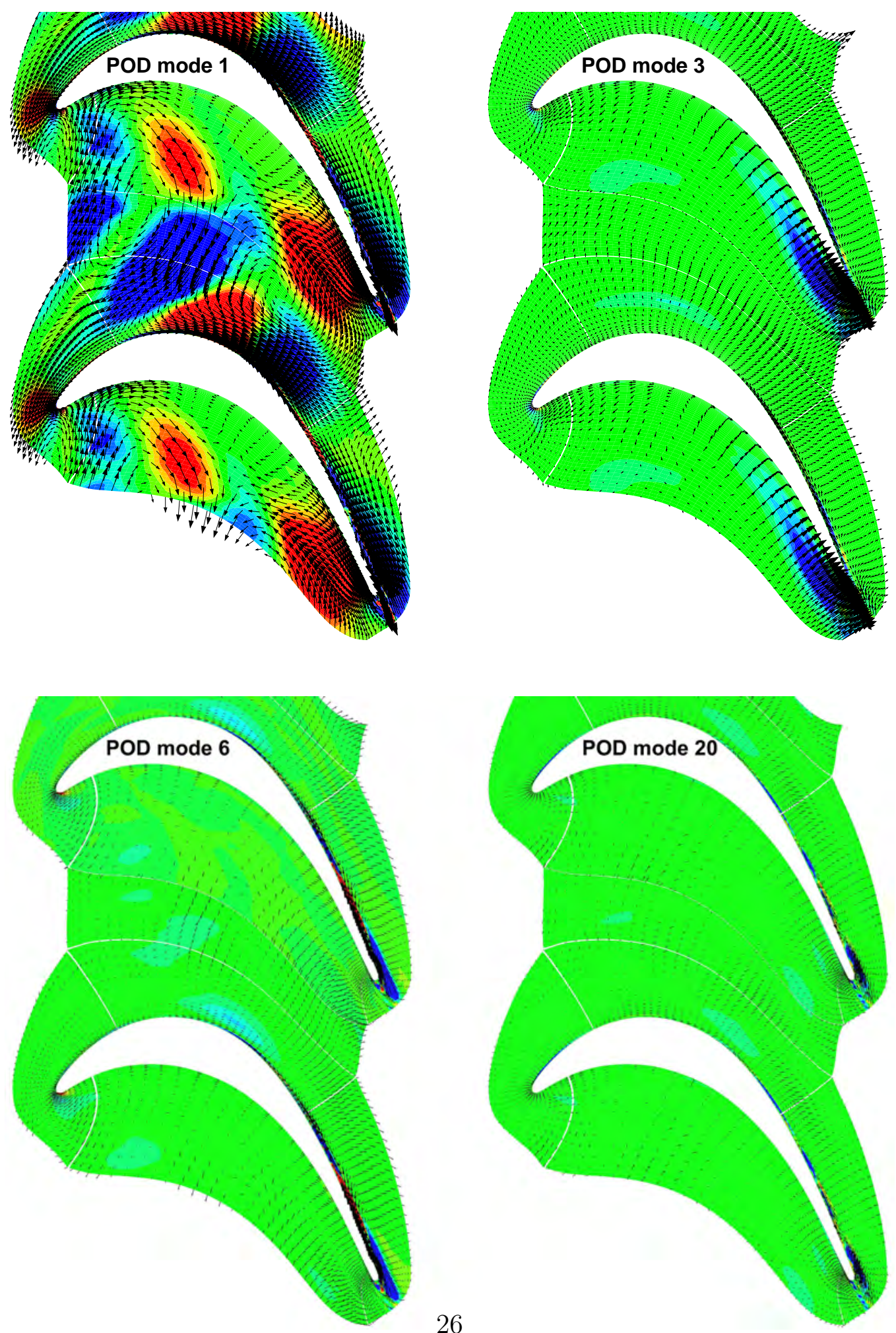

Figure 8: TKE production rate per POD mode. Red and blue color represent negative and positive TKE production rate, respectively 

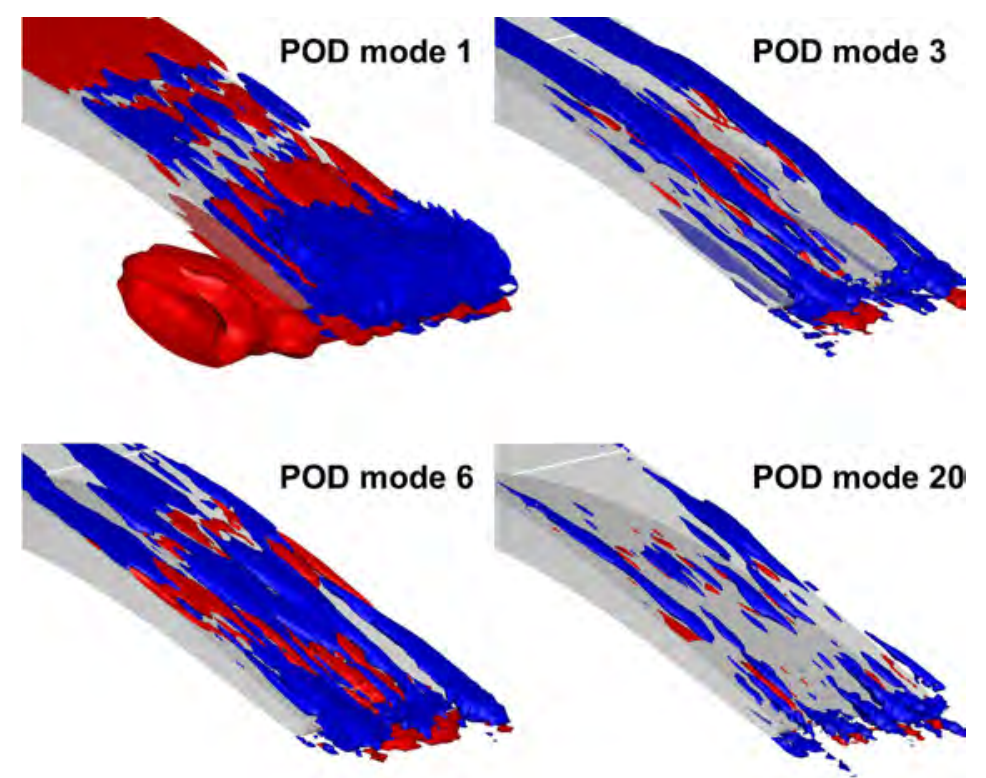

Figure 9: Isosurface of the TKE production rate per POD mode. Red and blue color represent negative and positive TKE production rate, respectively 
the other two terms will be discussed in the next section through low-order truncation.

The TKE production rate is shown in Figs. 8 as a 2D representation and in Fig. 9 as a 3D view on the rear part of the blade suction side. The first POD mode (and similarly the second one) generates the greatest amount of TKE inside the blade passage. The TKE production associated with this POD mode shows areas of positive and negative generation rate at the edges of the two large scale vortices generated in the passage during the wake migration. This mode clearly identifies zones where the turbulence is converted back to mean flow energy (red regions in the plot). This is similar to what occurs in a 2D inviscid flow, there the flux of energy from large to small scales must be accompanied by another compensating flux of energy back towards larger length scales (Doering and Gibbon, 1995). In the present case, back-scatter occurs only locally, since once integrated over the whole domain (as done in the next section) the energy flux is from the mean flow to the turbulence. This mechanism of TKE production is almost uniform in the spanwise direction as highlighted by the 3D view of Fig. 9. The following modes show that TKE is produced mainly in the rear part of the blade in the boundary layer regions. Effects of mode 3 are prevalently confined in the pressure side region, while modes 6 and 20 exhibit marked TKE production in the rear part of the blade suction side. For these modes the production of TKE occurs by means of 3D structures elongated in the streamwise direction along the blade surface, as previously observed. Also in this case local backscatter can be observed in between the elongated structures responsible for TKE production. The contribution to TKE production due to mode 20 into 
the boundary layer is evidently due to structures smaller than those observed in the same region in mode 6 , which is related to the large scales of the wake migrating into the potential flow region, as previously observed.

\subsection{Contribution to losses}

The spatial integration over the whole $3 \mathrm{D}$ cascade passage of the terms appearing in Eq. 6 for each POD mode provides the contribution of that mode to the overall entropy rate of change. However, a single POD mode is not able to completely trace a "structure" or particular dynamics. Hence, the contributions to the entropy rate of change of that dynamics can be identified accounting for the values of terms I, II and III of Eq. 6 due to different ensembles of POD modes that pertain and describe the same flow features. The different families can be identified by means of different criteria. Namely, the deterministic flow features, that are related to a particular frequency and its harmonic (e.g. the wake passing events previously described), are identified looking at the spectral analysis. Otherwise, the turbulent structures are not related to a particular frequency and several POD modes are required to identify such structures. The choice of a proper ranking (based on the loss production) is used to split between the turbulent structures carried by the wake and the finer scale structures isolated by low and high order modes, respectively.

The cumulative integral of the different terms appearing in Eq. 6 is provided in Fig. 10. The cumulative contribution of the kinetic energy is also shown in the plot for comparison. The gray line provides the overall entropy rate of change in the full 3D domain, the labels of the axis (on the left) 


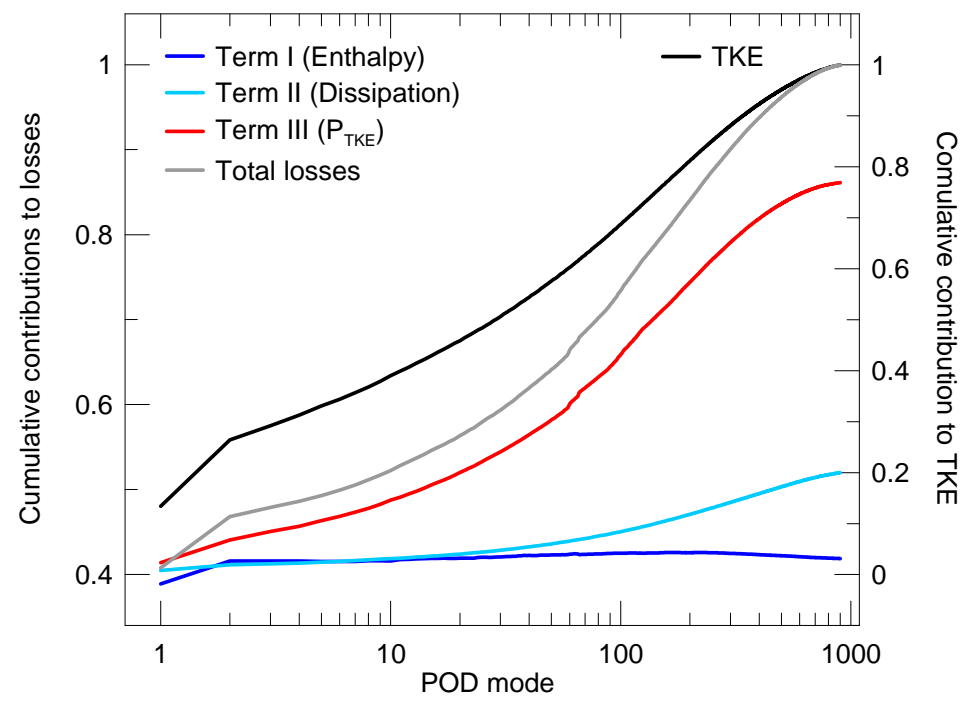

Figure 10: Cumulative contribution to the losses (entropy rate of change) of each POD mode. The values are obtained by integration over the 3D measurement domain. The legend refers to Eq. 6

start from the base level of $40 \%$ that corresponds to the integral of the viscous dissipation (term IV of eq. 6). The red line represents the cumulative contribution of the TKE production. This is evidently the dominant term when compared with the others, since it is responsible for almost $50 \%$ of the overall entropy rate of change. The dissipation term (light blue line) and the velocity-enthalpy correlation cover only slightly above $10 \%$ of the overall loss budget.

Looking at the different dynamics, the first 12 modes (excluding modes 3 to 5 as previously observed) describe the large scale deterministic structures due to wake migration, as identified from the Fourier analysis of their eigenvectors previously shown. The entropy rate of change related to these modes is about $13 \%$ of the total, while they capture about $35 \%$ of the TKE. 


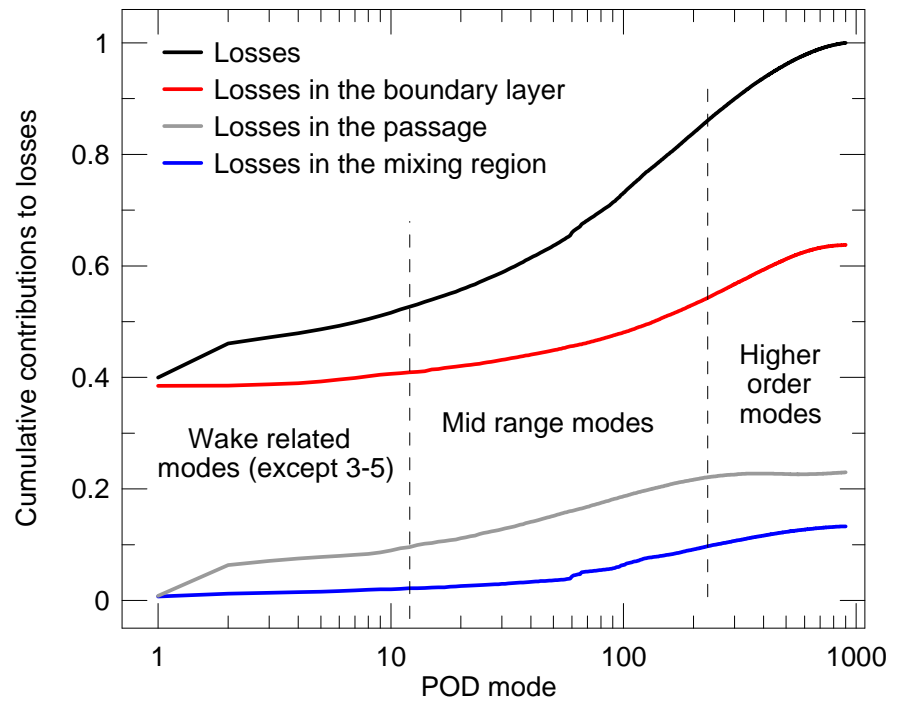

Figure 11: Cumulative contribution to the losses (entropy rate of change) of each POD mode. The values are obtained by integration over the 3D measurement domain.

It has to be noted that the contribution of the velocity-enthalpy correlation term is limited to the first two modes. The overall contribution due to velocity-enthalpy correlation is, in fact, determined by the fractions provided by summation of modes 1 and 2 . This has the important consequence that reduced order models aimed at describing the effects due to velocityenthalpy correlation can be constructed retaining (in the energy equation) just two POD modes (i.e. a Galerkin projection with only 2 modes provides almost $100 \%$ of the overall dynamics of the system). The dissipation term is negligible for these dynamics, and it becomes significant only after mode 20, according to what was shown in Lengani et al. (2016). The POD modes related to the large scale deterministic oscillations due to the passing wake contribute only marginally to the energy dissipation, due to the combination of viscosity and spatial derivative of the modes. Thus, a first important as- 
pect raised by the present analysis is that losses due to the propagation of the deterministic large scale structures attached to the wake are essentially due to the production of turbulence kinetic energy, with negligible viscous dissipation. Consequently, wake migration related losses are expected to not be influenced by the variation of the Reynolds number.

In order to better identify the dynamical features (i.e. POD mode family) and where (spatially) they act to produce the largest amount of losses a further analysis step has to be performed. Namely, the spatial integration of the entropy rate of change has been limited to different flow regions: the boundary layer region, the potential flow region of the blade passage, and the mixing region downstream of the trailing edge. The former has been defined by a threshold level on the vorticity. The mixing region has been bounded by lines normal to the flow direction at the blade trailing edge, while the remaining space of the passage represent the potential flow region. Fig. 1 provides a sketch of the different integration areas. The results are shown in Fig. 11 as a function of the POD modes. Two dashed vertical lines are superimposed to the plot to provide a distinction between the different families of POD modes as it will be explained in the followings.

The modes from 1 to 12 (the first vertical line), except modes 3 to 5 as previously observed, are related to the large scale deterministic structures of the wake. The losses obtained in the boundary layer region due to these modes give a direct estimation of the unsteady wake-boundary layer interaction process caused by the formation of deterministic structures as those identified in Fig. 6 for mode 6.

The second vertical line (at mode 220) is adopted to identify the modes 
that produce losses in the potential flow region but are not related to the deterministic events. This family of modes (named "mid range modes") has been identified looking at the loss production in the potential flow region (gray line) that is completely captured by modes below 220. In fact, above this mode, the gray line is flat indicating that no more losses are produced there. Hence, the modes between 13 and 220 are responsible for losses in the whole computational domain but are not related to deterministic events. These modes are representative of the turbulence, finer scales, carried by the wake such as the previously discussed mode 20. It is worth noting that these modes also trace streaky structures in the boundary layer, thus once spatial integration is limited to this flow region it provides the loss contribution due to the wake-boundary layer interaction process. Conversely, integration in the potential flow region further contribute to the entropy rate of change due to wake migration (now due to the finer stochastic structures embedded in the bulk of the wake).

The higher modes (above 220) are related to the smaller scale structures that can be identified just within the boundary layer and in the mixing region. These modes do not contribute to losses in the blade passage (as will be clearer from looking at Fig. 12 in the following). They are consequently unrelated to the structures (deterministic or stochastic) carried by the wake and are attributed to the finer scale structures forming in the boundary layer as a consequence of the transition process.

Summarizing, the wake migrating losses in the blade passage are isolated by the modes related to the deterministic large scale structures (modes 1 to 12) plus the finer scales (modes 13 to 220) restricted to the potential flow 
region. These losses are about $23 \%$ of the total losses, and the modes related to the large scale structures of the wake contribute about $10 \%$ of the total losses there, the rest has to be attributed to the "mid range" POD modes (up to 220). These losses are due to the finer structures embedded within the bulk of the wake (that have no significant amplitudes in the spectra) and contribute to the $13 \%$ of loss produced into the LPT passage during wake migration.

The large scale wake related modes partially contribute to the generation of TKE in the boundary layer (red line). This contribution is only about $2 \%$ of the total but it has to be observed that the first POD mode has a negative contribution to the loss generation rate. The remainder of the losses generated within the boundary layer are due to smaller scale structures (higher order POD modes); they represent about 25\% of the total losses and are about equally split between the "mid range" and "higher order" POD modes. The remaining $12 \%$ of the loss budget is generated in the mixing region, just downstream of the blade trailing edge. The first 50 modes, the largest scale dynamics, do not contribute to the generation of losses in this region.

In order to further emphasize the capability of the procedure proposed here, the spatial distribution of the rate of change of entropy due to turbulent kinetic energy production and finer scales viscous dissipation (terms II and III of Eq. 6) are reported in Fig. 12 and 13 separating the loss contribution of the three families of POD modes previously identified from Fig. 11. The $2 \mathrm{D}$ view only is presented in Fig. 12 and shows that the loss generation due to the large scale structures of the wake (plot on left) occurs within the 

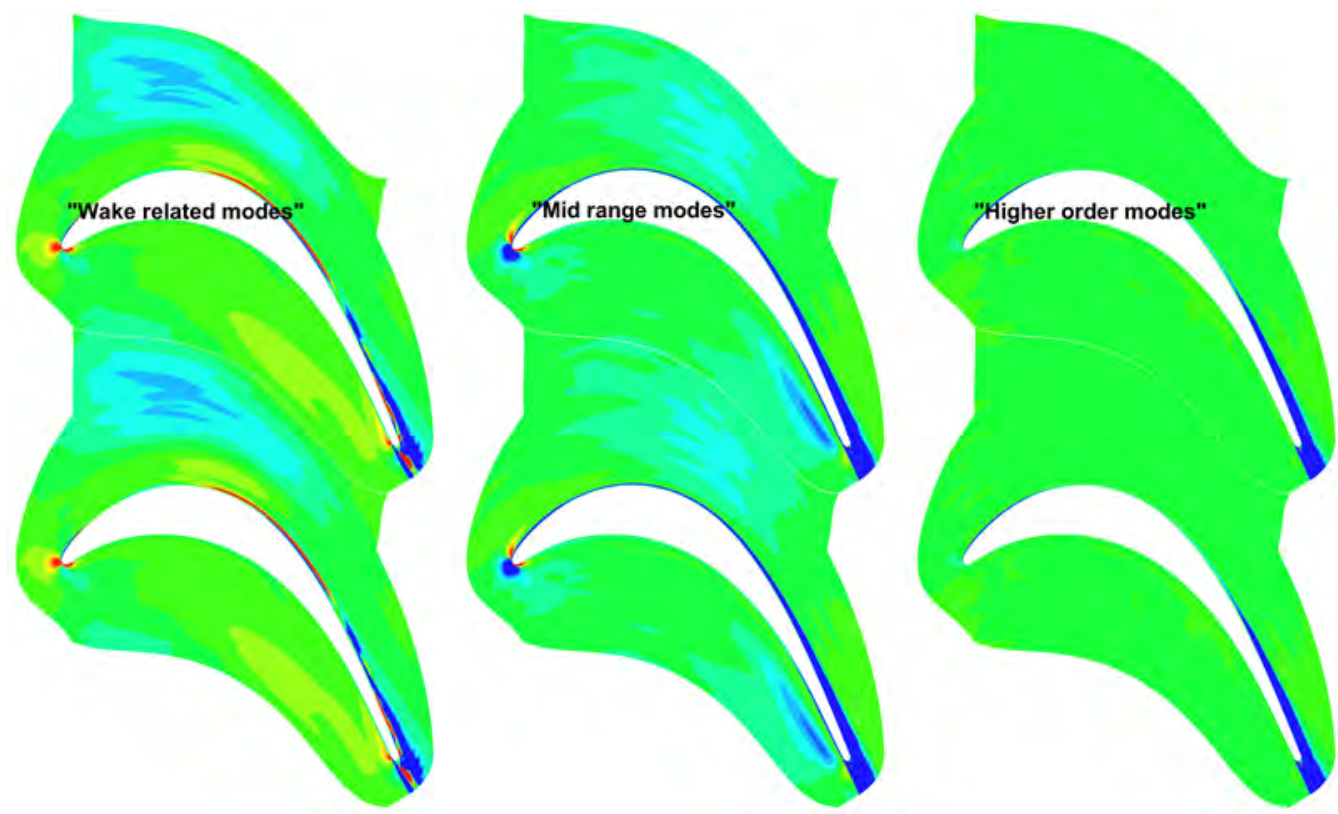

Figure 12: Entropy rate of change associated to the term III of eq. 6 .

passage (cyan region in the plot), as well as in the rear part of the suction side. The region of loss generation in the fore part of the potential flow region of the blade passage is observed where the phenomena of stretching and bowing of the wake are more pronounced. The loss production within the boundary layer observed behind the peak suction is instead related to the interaction of the large scale structures of the wake and the decelerating boundary layer. The highest values can be observed in the rear part of the blade suction side, where the intermittent bypass-like transition is forced by the migrating wake. The loss generation due to the "mid range mode" (plot in the middle) is related to the boundary layer developing on the entire pressure and suction sides and to the finer structures carried by the passing wakes during migration in the blade passage (see again the cyan region at mid chord in the plot on the center). The turbulence carried by the wake, 
that has a stochastic nature, is captured by the mid range order POD modes and, as previously observed, contributes significantly to the losses generated in the blade passage. The remaining higher order POD modes (plot on the right) induce losses mainly in the decelerating part of the blade suction side. Indeed, the finer scale structures growing into the boundary layer (traced by the higher order, less energetic, POD modes) are responsible for the high level of entropy rate of change observable in the wall proximity on this plot in the rear suction-side region. These are not related to wake events (either deterministic nor stochastic) and are consequently attributed to the turbulence produced by the spontaneous transition process occurring in the rear suction-side boundary layer, where the viscous term (term IV of eq. 6) is locally lower than the term of production of TKE.

Fig. 13 shows the resolved scale dissipation (term II of Eq.6) for the three families of modes. This term is always confined to a region close to the wall. This term is very small for the large scale wake related modes (plot on left). It is mainly due to the finer structures generated during the wake-boundary layer interaction along the blade suction side (mid range modes) as well as to the small scale structures generated due to the transition process (higher order modes). It is worth to note that the contribution of this term in the potential flow region of the passage is negligible for all the three dynamics, and especially for both the deterministic and stochastic wake related modes. This provides further evidence that energy dissipation due to the wake migration process is essentially due to the production of TKE, since viscous effects are negligible outside the boundary layer. 

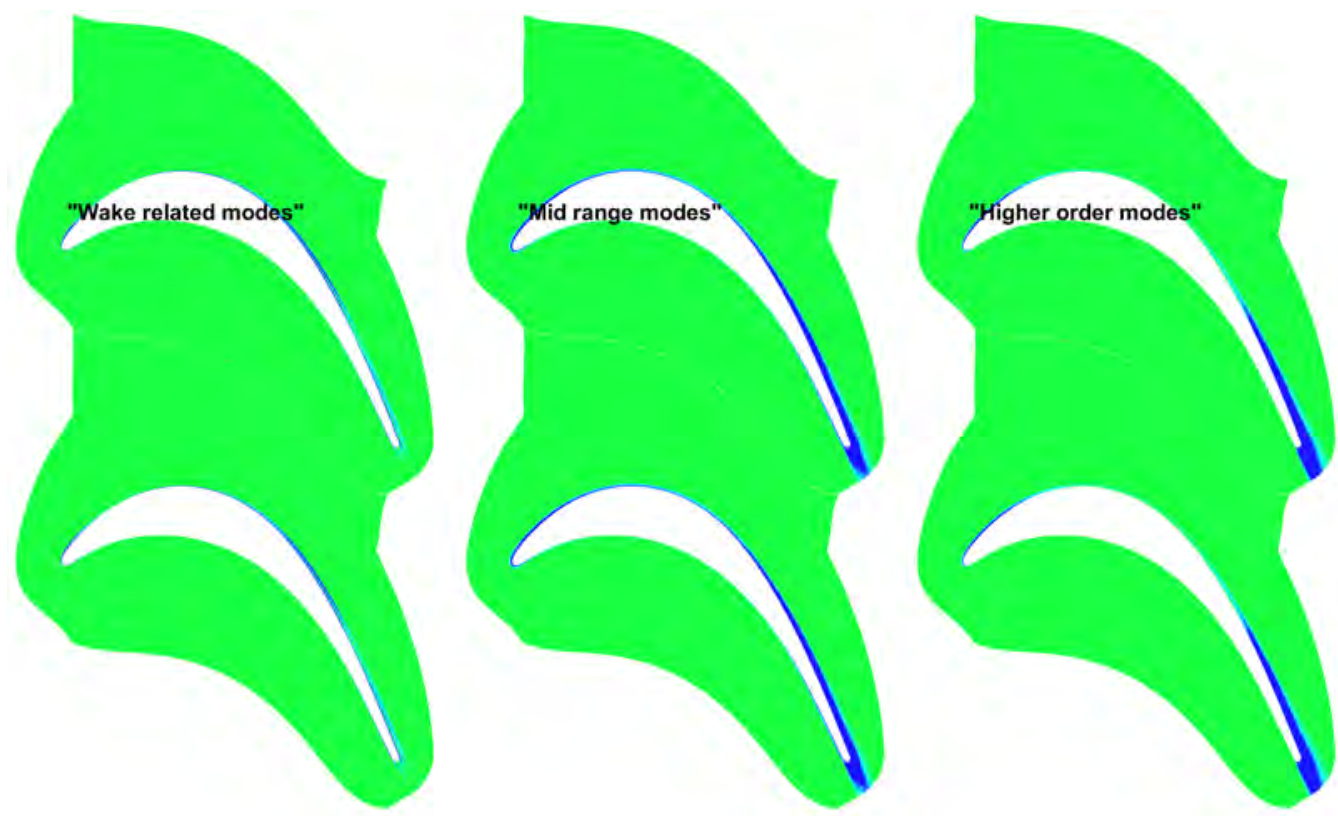

Figure 13: Entropy rate of change associated to the term II of eq. 6.

\section{Conclusions}

Large datasets, such as time resolved LES of unsteady flow fields, require considerable efforts for their interpretation. In the present paper, POD has been applied to high fidelity LES data of a low pressure turbine blade perturbed by unsteady passing wakes in order to characterize and split the different phenomena and dynamics responsible for loss generation. The procedure makes use of the bi-orthogonality condition of POD modes and related temporal coefficients to evaluate the entropy rate of change corresponding to each POD mode.

The procedure has been shown to be capable of identifying the different phenomena generating the complex unsteady flow field: e.g. the large scale deterministic structures attached to the leading and trailing boundaries of 
the wake, the finer stochastic scales carried in the bulk of the wake, as well as the smaller streamwise oriented scale generating in the boundary layer as a consequence of the bypass-like transition process induced by wakes.

The different terms that contribute to the entropy rate of change have been evaluated separately, projecting the velocity and enthalpy fields onto the orthogonal basis spanned by the temporal coefficient obtained by the kinematic kernel. The production of turbulent kinetic energy is the most relevant term to loss generation. The first modes capture the loss increase due to the large scale deterministic structures of the wake migrating into the potential flow region, and highlight that it occurs mainly in the fore part of the blade passage where the wakes are dilated, stretched and bowed. Also the finer resolved scales carried in the bulk of the wake contribute significantly to the generation of entropy in the passage. The dissipation term and velocity-enthalpy correlation cover only slightly above $10 \%$ of the overall loss budget. The dissipation is confined mainly to within the boundary layer, with negligible contribution due to the large scale structure carried by the wake. On the other hand, the velocity-enthalpy correlation term is captured in its entirety by just the first two POD modes.

The entropy rate of change has been quantified in properly defined control volumes and discerning between families of POD modes it has been possible to provide information on where the loss source comes from, and by which dynamical feature it is caused. Particularly, it has been observed that the first 12 modes contribute significantly just to the loss generation rate within the blade passage and partially to that in the boundary layer. The losses in the boundary layer are instead caused by 2 different dynamics. The first 
cause is connected to the turbulence carried by the passing wakes since the mid range POD modes identify structures that coexist in the passage and in the boundary layer. The smaller scale structures that occur just within the transitional and turbulent boundary layer in the rear part of the suction side are the second cause (identified by the higher order POD modes). These two mechanisms contribute roughly equally to the losses generated in the boundary layer. Hence, the loss contribution from a large dataset obtained from laboratory or numerical experiments of a complex 3D unsteady flow field may be easily extracted by using the proposed post-processing procedure, also in other applications.

The authors would like to thank General Electric Global Research for permission to publish this work. Computations have been conducted on the UK national facility ARCHER using resources provided by the UK turbulence consortium under Grant No. EP/L000261/1.

Adrian, R. J., 2007. Hairpin vortex organization in wall turbulence. Phys. Fluids 19, 041301-1-041301-16.

Alam, M., Sandham, N., 2000. Direct numerical simulation of 'short' laminar separation bubbles with turbulent reattachment. J. Fluid Mech. 410, 1-28.

Binder, A., Förster, W., Kruse, H., Rogge, H., 1985. Experimental investigation into the effect of wakes on the unsteady turbine rotor flow. ASME Journal of Engineering for Gas Turbines and Power 107 (2), 458-465.

Binder, A., Schröder, T., Hourmouziadis, J., April 1989. Turbulence measurements in a multistage low-pressure turbine. J Turbomach 111 (2), 153-161. 
Borée, J., 2003. Extended proper orthogonal decomposition: a tool to analyse correlated events in turbulent flows. Experiments in fluids 35 (2), 188-192.

Bourgeois, J., Noack, B., Martinuzzi, R., 2013. Generalized phase average with applications to sensor-based flow estimation of the wall-mounted square cylinder wake. J. Fluid Mech. 736, 316-350.

Coull, J. D., Hodson, H. P., 2011. Unsteady boundary-layer transition in low-pressure turbines. J. Fluid Mech. 681, 370-410.

Denton, J. D., 1993. Loss mechanisms in turbomachines. J. Turbomach. 115 (4), 621-656.

Dick, E., Kubacki, S., 2017. Transition models for turbomachinery boundary layer flows: a review. International Journal of Turbomachinery, Propulsion and Power $2(2), 4$.

Doering, C. R., Gibbon, J. D., 1995. Applied analysis of the Navier-Stokes equations. Vol. 12. Cambridge University Press.

Hanjalić, K., Launder, B., 2011. Modelling turbulence in engineering and the environment: second-moment routes to closure. Cambridge university press.

Hodson, H. P., Howell, R. J., 2005. Bladerow interactions, transition, and high-lift aerofoils in low-pressure turbines. Annu. Rev. Fluid Mech. 37, 71-98.

Hussain, A., Reynolds, W., 1970. The mechanics of an organized wave in turbulent shear flow. J. Fluid Mech. 41, 241-258. 
Jones, L., Sandberg, R., Sandham, N., 2008. Direct numerical simulations of forced and unforced separation bubbles on an airfoil at incidence. J. Fluid Mech. 602, 175-207.

Kennedy, C. A., Carpenter, M. H., Lewis, R. M., 2000. Low-storage, explicit runge-kutta schemes for the compressible navier-stokes equations. Applied numerical mathematics 35 (3), 177-219.

Kennedy, C. A., Gruber, A., 2008. Reduced aliasing formulations of the convective terms within the navier-stokes equations for a compressible fluid. Journal of Computational Physics 227 (3), 1676-1700.

Kim, J. W., Sandberg, R. D., 2012. Efficient parallel computing with a compact finite difference scheme. Computers \& Fluids 58, 70-87.

Kurelek, J. W., Lambert, A. R., Yarusevych, S., 2016. Coherent structures in the transition process of a laminar separation bubble. AIAA Journal, $1-15$.

Lacarelle, A., Faustmann, T., Greenblatt, D., Paschereit, C. O., Lehmann, O., Luchtenburg, D. M., Noack, B. R., 2009. Spatiotemporal characterization of a conical swirler flow field under strong forcing. J. Eng Gas Turb Power 131, 031504 (1-12).

Legrand, M., Nogueira, J., Lecuona, A., 2011a. Flow temporal reconstruction from non-time-resolved data part I: mathematic fundamentals. Exp. Fluids 51 (4), 1047-1055.

Legrand, M., Nogueira, J., Tachibana, S., Lecuona, A., Nauri, S., 2011b. 
Flow temporal reconstruction from non-time-resolved data part II: practical implementation, methodology validation, and applications. Exp. Fluids $51(4), 861-870$.

Lengani, D., Simoni, D., Ubaldi, M., Zunino, P., Bertini, F., 2017a. Analysis of the reynolds stress component production in a laminar separation bubble. International Journal of Heat and Fluid Flow 64, 112-119.

Lengani, D., Simoni, D., Ubaldi, M., Zunino, P., Bertini, F., 2017b. Coherent structures formation during wake-boundary layer interaction on a LP turbine blade. Flow, Turbulence and Combustion 98 (1), 57-81.

Lengani, D., Simoni, D., Ubaldi, M., Zunino, P., Guida, R., 2016. Turbulence production, dissipation and length scales in laminar separation bubbles. ETMM11, 1-6.

Leschziner, M., 2015. Statistical turbulence modelling for fluid dynamicsdemystified: an introductory text for graduate engineering students. World Scientific.

Liu, Z., Adrian, R. J., Hanratty, T. J., 2001. Large-scale modes of turbulent channel flow: transport and structure. J. Fluid Mech. 448, 53-80.

Lumley, J. L., 1967. The structure of inhomogeneous turbulent flows. A.M. Yaglom, V.I. Tatarski (Eds.), Atmospheric Turbulence and Wave Propagation, 166-178.

Michelassi, V., Chen, L., Pichler, R., Sandberg, R. D., 2015. Compressible direct numerical simulation of low-pressure turbines-part II: Effect of inflow disturbances. J. Turbomach. 137 (7), 071005 1-12. 
Michelassi, V., Chen, L., Pichler, R., Sandberg, R. D., Bhaskaran, R., 2016. High-fidelity simulations of low-pressure turbines: Effect of flow coefficient and reduced frequency on losses. J. Turbomach. 138 (11), 111006.

Michelassi, V., Wissink, J. G., 2015. Turbulent kinetic energy production in the vane of a low-pressure linear turbine cascade with incoming wakes. International Journal of Rotating Machinery 2015.

Michelassi, V., Wissink, J. G., Fröhlich, J., Rodi, W., 2003. Large-eddy simulation of flow around low-pressure turbine blade with incoming wakes. AIAA journal 41 (11), 2143-2156.

Nagabhushana Rao, V., Tucker, P. G., Jefferson-Loveday, R. J., Coull, J. D., 2013. Large eddy simulations in low-pressure turbines: Effect of wakes at elevated free-stream turbulence. Int. J. Heat Fluid Flow 43, 85-95.

Nicoud, F., Ducros, F., 1999. Subgrid-scale stress modelling based on the square of the velocity gradient tensor. Flow, turbulence and Combustion $62(3), 183-200$.

Perrin, R., Cid, E., Cazin, S., Sevrain, A., Braza, M., Moradei, F., Harran, G., 2007. Phase-averaged measurements of the turbulence properties in the near wake of a circular cylinder at high reynolds number by $2 \mathrm{C}-\mathrm{PIV}$ and 3C-PIV. Exp Fluids 42, 93-109.

Praisner, T. J., Clark, J. P., Nash, T. C., Rice, M. J., Grover, E. A., 2006. Performance impacts due to wake mixing in axial-flow turbomachinery. In: ASME Turbo Expo 2006: Power for Land, Sea, and Air. pp. 1821-1830. 
Rogers, M. M., 2002. The evolution of strained turbulent plane wakes. J. Fluid Mech. 463, 53-120.

Sandberg, R. D., Michelassi, V., Pichler, R., Chen, L., Johnstone, R., 2015. Compressible direct numerical simulation of low-pressure turbinespart I: Methodology. J. Turbomach. 137 (5), 051011.

Sarkar, S., 2008. Identification of flow structures on a LP turbine blade due to periodic passing wakes. J. Fluid Eng - T ASME 130, 061103 (10 pages).

Sarkar, S., Voke, P., 2006. Large-eddy simulation of unsteady surface pressure overa low-pressure turbine blade due to interactions of passing wakes and inflexional boundary layer. J. Turbomach. 128, 221-231.

Satta, F., Simoni, D., Ubaldi, M., Zunino, P., Bertini, F., 2014. Loading distribution effects on separated flow transition of ultra-high-lift turbine blades. AIAA J. of Prop. and Power 30, 845-856.

Simoni, D., Ubaldi, M., Zunino, P., 2013. Experimental investigation of the interaction between incoming wakes and instability mechanisms in a laminar separation bubble. Exp. Therm. Fluid Sci 50, 54-60.

Sirovich, L., 1987. Turbulence and the dynamics of coherent structures. part I-III. Q Appl Math 45, 561-590.

Stieger, R. D., Hodson, H., 2004. The transition mechanism of highly loaded low-pressure turbine blades. J. Turbomach. 126, 536-543.

Stieger, R. D., Hodson, H. P., 2005. The unsteady development of a turbu- 
lent wake through a downstream low-pressure turbine blade passage. J. Turbomach. 127, 388-394.

van de Wall, A. G., Kadambi, J. R., Adamczyk, J. J., 2000. A transport model for the deterministic stresses associated with turbomachinery blade row interactions. J. Turbomach. 122 (4), 593-603.

van Oudheusden, B. W., Scarano, F., van Hinsberg, N. P., Watt, D. W., 2005. Phase-resolved characterization of vortex shedding in the near wake of a square-section cylinder at incidence. Exp. Fluids 39, 86-98.

Wen, X., Tang, H., Duan, F., 2016. Interaction of in-line twin synthetic jets with a separated flow. Physics of Fluids 28 (4), 043602.

Wheeler, A. P. S., Sandberg, R. D., Sandham, N. D., Pichler, R., Michelassi, V., Laskowski, G., 2016. Direct numerical simulations of a high-pressure turbine vane. J. Turbomach. 138 (7), 071003.

Wu, X., Durbin, P. A., 2001. Evidence of longitudinal vortices evolved from distorted wakes in a turbine passage. J. Fluid Mech. 446, 199-228.

Wu, X., Jacobs, R. G., Hunt, J. C. R., Durbin, P. A., 1999. Simulation of boundary layer transition induced by periodically passing wakes. J. Fluid Mech. 398, 109-153.

Yarusevych, S., Kotsonis, M., 2017. Effect of local DBD plasma actuation on transition in a laminar separation bubble. Flow, Turbulence and Combustion 98 (1), 195-216. 
Zaki, T. A., Wissink, J. G., Durbin, P. A., Rodi, W., 2009. Direct computations of boundary layers distorted by migrating wakes in a linear compressor cascade. Flow, turbulence and combustion 83 (3), 307-322. 


\section{University Library}

\section{- M M N E R VA A gateway to Melbourne's research publications}

Minerva Access is the Institutional Repository of The University of Melbourne

Author/s:

Lengani, D;Simoni, D;Pichler, R;Sandberg, RD;Michelassi, V;Bertini, F

Title:

Identification and quantification of losses in a LPT cascade by POD applied to LES data

Date:

2018-04-01

Citation:

Lengani, D., Simoni, D., Pichler, R., Sandberg, R. D., Michelassi, V. \& Bertini, F. (2018).

Identification and quantification of losses in a LPT cascade by POD applied to LES

data. International Journal of Heat and Fluid Flow, 70, pp.28-40. https://doi.org/10.1016/

j.jheatfluidflow.2018.01.011.

Persistent Link:

http://hdl.handle.net/11343/241855 